\title{
Landshut Univeristy of Applied Sciences Holding ISBN: 9788366675599
}

Hochschule für angewandte Wissenschaften Landshut

Am Lurzenhof 1, 84036 Landshut, Germany

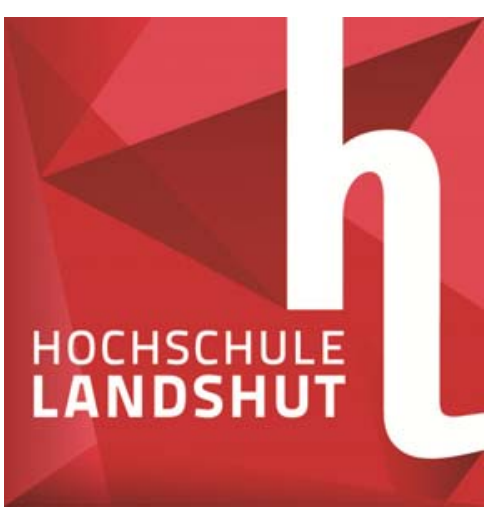

Library Holding:

Katalog der Hochschulbibliothek Landshut

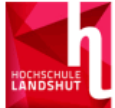

HOCHSCHULE LANDSHUT

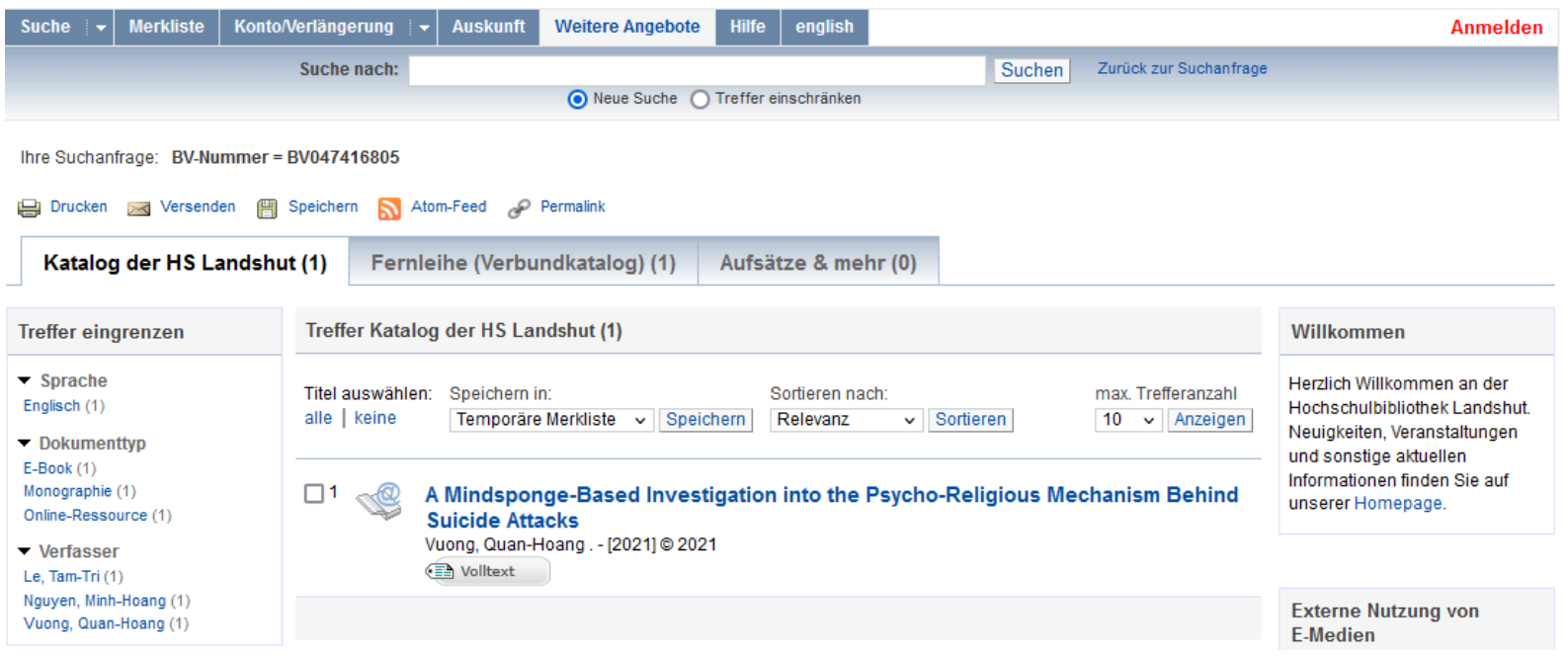




\section{Katalog der Hochschulbibliothek Landshut}

\begin{tabular}{r|r|r|r|r|r|r|} 
Suche & Merkliste & Konto/Verlängerung & Anmelden \\
\hline Suche nach: & Auskunft & Weitere Angebote & Hilfe & english & Suchen Zurück zur Suchanfrage \\
\hline
\end{tabular}

Zurück zur Trefferliste

믑 Drucken Versenden 謂 Speichern Permalink

Katalog der HS Landshut (1/1)

Externe Nutzung von E-Medien

Speichern in:

Temporäre Merkliste $\vee$ Speichern

A Mindsponge-Based Investigation into the Psycho-Religious Mechanism Behind Suicide Attacks

Volltext

Verfasser: Vuong, Quan-Hoang

Erscheinungsort: Warsaw ; Berlin

Verlag: Sciendo

Erscheinungsjahr: [2021]

Umfang: 1 online resource (127 pages)

ISBN: 9788366675599

Fehlerhaften Link bitte unter 'Verfügbarkeit' melden!

\begin{tabular}{|c|c|c|}
\hline Exemplare & Verfügbarkeit & mehr zum Titel \\
\hline Titel: & A Mindsponge- $\mathrm{B}$ & ed Investigation into the Psycho-Religious Mechanism Behind Suicide Attacks \\
\hline Von: & Quan-Hoang Vu & g, Minh-Hoang Nguyen, Tam-Tri Le \\
\hline Verfasser: & Vuong, Quan-Ho & \\
\hline Verf./Hrsg./Bet.: & Le, Tam-Tri & \\
\hline ...: & Verfasser & \\
\hline Verf./Hrsg./Bet.: & Nguyen, Minh-H & \\
\hline ...: & Verfasser & \\
\hline Erscheinungsort: & Warsaw ; Berlin & \\
\hline Verlag: & Sciendo & \\
\hline Erscheinungsjahr: & : [2021] & \\
\hline Erscheinungsjahr: & : ๑ 2021 & \\
\hline Umfang: & 1 online resourc & 127 pages) \\
\hline Bemerkung: & Description base & on online resource; title from PDF title page (publisher's Web site, viewed 29. Jul 2021) \\
\hline Bemerkung: & In English & \\
\hline ISBN: & 9788366675599 & \\
\hline Abstract: & $\begin{array}{l}\text { The book examir } \\
\text { employs the min } \\
\text { investigations us } \\
\text { strategists in the }\end{array}$ & $\begin{array}{l}\text { s the psycho-religious mechanism behind the violent extremism of suicide attacks in the post-9/11 world. It } \\
\text { ponge concept, an original dataset, and original research results obtained from the authors' statistical } \\
\text { g the Hamiltonian Markov chain Monte Carlo technique. It provides insights and implications for policymakers and } \\
\text { efforts to engage in peace talks and reduce violent conflicts worldwide }\end{array}$ \\
\hline Volltext E-Book: & https://www.degr & ter.com/isbn/9788366675599 \\
\hline DOI: & $10.2478 / 978836$ & 75599 \\
\hline Volltext : & https://www.degr & ter.com/isbn/9788366675599 \\
\hline ISBN and. Ausg.: & 9788366675582 & \\
\hline B3Kat-ID: & BV047416805 & \\
\hline Aufnahme am: & 13.08 .2021 & \\
\hline Änderung am: & 14.09.2021 & \\
\hline
\end{tabular}

Hochschulangehörige können E-Medien auch außerhalb vom Campus öffnen, Link zum Katalog für Hochschulangehörige und weitere Infos

Entliehen bis 31.12.9999?

Bücher mit Leihfrist 31.12.9999 bitte vormerken. Wir stellen den gewünschten Titel so schnell wie möglich für Sie bereit. 
*Permalink:

https://opac.haw-

landshut.de/TouchPoint/singleHit.do?methodToCall=showHit\&curPos=1\&identifier=2 SOLR SE

RVER 809752209

(Accessed: September 19, 2021) 